\title{
Vectorial Characterisation of Electromagnetic Fields by Infrared Thermography
}

\author{
by P. Levesque, L. Leylekian, and D. Balageas
}

ONERA, BP 72, 92320 Châtillon cedex, France, E-mail: levesque@onera.fr, leylekian@onera.fr, balageas@onera.fr

\begin{abstract}
:
EMIR technique is now able to measure the polarisation of EM fields, thanks to highly anisotropic photothermal films. The films and the numerical model used to evaluate their electromagnetic behaviour are described. Based on the use of a model, a thermal optimisation of the sensor, taking into account the amplitude modulation frequency, is presented. The technique is applied to the analysis of the EM field, at $12 \mathrm{GHz}$, in the vicinity of the end of a wave-guide. Validation is achieved through comparison of the experimental results to theory.
\end{abstract}

\section{Introduction}

ONERA is developing and improving the EMIR (ElectroMagnetic InfraRed) method allowing to visualise EM (electromagnetic) fields [1]. This method, mainly used for non destructive evaluation of dielectric or absorbing materials, antenna radiation pattern characterisation and mapping of the electric field around objects or inside cavities, consists in measuring by means of an IR camera the temperature increase of a thin photothermal film, which is proportional to the intensity of the electric field component tangential to the film [2]. In order to avoid the distortion of the temperature field due to the natural convection, and to increase the spatial resolution, the amplitude of the EM field is modulated at a low frequency (some $\mathrm{Hz}$ ), and a lock-in thermographic system is used [3].

Due to the physical principle of the photon-heat conversion, only the squared magnitude (intensity) of the electric field is directly measured, but the use of microwave holography and the combination of several intensity images allow to obtain relative phase image $[4,5]$, with the restriction that the EM fields have just one component in the plane of the film. Otherwise, a pseudo scalar phase is evaluated.

In order to extend the field of application of EMIR method, the feasibility of measuring the polarisation of the EM fields is studied. This is possible thanks to the development of a new type of photothermal film, highly non-isotropic, which is described in the first part of the paper. A numerical model is used to optimise the electromagnetic behaviour of the films. The thermal optimisation of the film, taking into account the space the amplitude modulation frequency is also presented.

The technique is validated by comparing the experimental results to numerical calculations in the analysis of the EM field, at $12 \mathrm{GHz}$, in the vicinity of the end of a waveguide.

\section{Electrical optimisation of the anisotropic photothermal film}

An anisotropic photothermal film is constituted by a thin dielectric film partially coated with a very thin electrical conducting coating (see Fig. 1). The coating is deposited following a pattern of parallel, rectilinear strips. These strips, absorbing partially the incident 
electromagnetic field, are characterised by their square resistance $R$, reciprocal of the product of their electrical conductivity by their thickness, and by geometrical parameters: space period $\Pi$ and fill factor $\phi$, ratio of their width $w$ to the space period. Such a grating is preferentially absorbing the component of the electrical field parallel to the strips. The system works like a polariser, but is used for its absorption and not for its transmission as commonly used. The anisotropy factor, ratio of the energies of the two components (parallel and perpendicular to the strips) is highly depending on these parameters $R, \phi$, and $\Pi$. To optimise the grid, we have used a code, based on the work of Boutiché and Petit [6], developed by the Laboratoire d'Optique Electromagnétique of Marseille. The code allows evaluating the absorption $A$, reflection $R$ and transmission $T$ of such gratings. Based on more than 30000 calculations, a data base was constituted and is presented here in Figure 2 under the form of a graph depending on the three parameters : $\phi, R, \lambda / \Pi, \lambda$ being the wavelength of the microwave. The graph presents the product of the transmissions for the two components of the field, $T_{H / /} T_{E / /}$ versus the ratio of their absorption, $A_{H / /} / A_{E / /}$ (the anisotropy factor). For a given frequency of the wave and a given space period, one can chose the fill factor and the square resistance to obtain a high anisotropy factor with a good transmission.

For validating these numerical results, experimental data are presented in Figure 3 , corresponding to an anisotropic film of $R=1020 \Omega, \phi=0,219$ and $\Pi=800 \mu \mathrm{m}$. These five infrared images correspond successively to: i) isotropic film heating, ii) anisotropic film, with consecutively: the field parallel to the strips, perpendicular to strips, the absorptivity and anisotropy factor distributions. For the area delimited by a circle, the mean values of these two last parameters are respectively 0.086 and 19 , which compare favourably to the calculated values of 0.086 and 15

\section{Thermal optimisation of the non isotropic photothermal film}

Let us consider the anisotropic photothermal film previously defined, with the parameters $R=1020 \Omega, w=175 \mu \mathrm{m}, \Pi=800 \mu \mathrm{m}$, and $\phi=175 / 800=0.219$. Taking into account the radiatoconvective heat losses, the theoretical temperature increase of the film can be calculated, considering the surface heat sources corresponding to the absorbing strips. The power generated in the strip has a density $P^{\prime}=P_{i n c} A(\phi, R, \lambda / \Pi), P_{i n c}$ being the incident power density and $A$ the absorptivity calculated by the electromagnetic code described previously.

The steady-state (no modulation) temperature increase of a uniform isotropic film $(\phi=1)$ is $T=P_{0} / 2 h=P_{i n c}\left(4^{*} 377 / R\right) /(2+377 / R)^{2}$. The distribution of temperature ${ }^{1}$ increase of the anisotropic film, in the $x$ direction perpendicular to the strip, presented in figure 4 , is given by the following formulas:

- in the absorbing strips $(-w / 2<x<w / 2)$ :

$$
\mathrm{T}(\mathrm{x}, \omega) /\left[\mathrm{P}^{\prime}{ }_{0} / 2 \mathrm{~h}\right]=[1-\operatorname{sh}(\Pi \cdot[1-\phi] \cdot \mathrm{q}(\omega) / 2) \cdot \operatorname{ch}(\mathrm{q}(\omega) \cdot \mathrm{x}) / \operatorname{sh}(\Pi \cdot \mathrm{q}(\omega) / 2)] /\left[1-\mathrm{i} \omega / \omega_{\mathrm{c}}\right]
$$

- in the non absorbing strips $(-\Pi / 2<x<-w / 2$ and $w / 2<x<\Pi / 2)$

$$
\mathrm{T}(\mathrm{x}, \omega) /\left[\mathrm{P}_{0}{ }_{0} / 2 \mathrm{~h}\right]=[\operatorname{sh}(\Pi \cdot \phi \cdot \mathrm{q}(\omega) / 2) \cdot \operatorname{ch}(\mathrm{q}(\omega) \cdot[\Pi / 2-|\mathrm{x}|])] /\left[\left(1-\mathrm{i} \omega / \omega_{\mathrm{c}}\right) \cdot \operatorname{sh}(\Pi \cdot \mathrm{q}(\omega) / 2)\right]
$$

with $\omega_{c}=2 h / \rho C e$, and $q(\omega)^{2}=\left(\omega_{c}-i \omega\right) / \kappa$, where $\kappa$ and $(\rho C)$ are respectively the diffusivity and the volume heat capacity of the film

\footnotetext{
1 The temperature field is time modulated, then in the presentation of the results, the parameter called temperature will be, for sake of simplicity, the amplitude, or module, of the modulated part of the temperature increase given by fomulas (1) and (2). This field is space modulated too due to the strips. For the same reason, we will call here $\mathrm{T}^{+}$the maximum of the amplitude of the modulated part of the temperature increase (center line of the absorbing strips) and $\mathrm{T}^{-}$its minimum (centerline of the non absorbing strips)
} 
The evaluation has been conducted for six values of the modulation frequency. As shown in Figure 4 the temperature distribution does not reproduce the exact square-shaped distribution of the absorbing strips. This could be achieved for frequencies higher than 100 $\mathrm{Hz}$ when the heat diffusion become really negligible at this geometrical scale, but increasing the modulation frequency leads to a loss of the film sensitivity (1/f law). The importance of these effects is shown in Table I, in particular by the ratio $\left(T^{+} / T\right)$ and the space modulation rate $2\left(T^{+}-T\right) /\left(T^{+}+T\right)$. The frequency of $10 \mathrm{~Hz}$ appears to be a good compromise.

On this graph, the temperature of the film is also given for the following limit cases: $i)$ no modulation $(f=0)$ and no heat diffusion in the film, leading to a temperature of the absorbing strip higher than that of the isotropic film: $T=P^{\prime} d / 2 h=0,39 P_{d} / 2 h$, with no heating for the non absorbing strips, ii) no modulation and infinite thermal conductivity for the film, leading to a temperature uniform on the whole film and equal to $T=0,39 \phi P_{0} / 2 h=0,086 P_{0} / 2 h$. In reality, the film thermal conductivity is finite and the film temperature is slightly modulated in space, with a mean (integrated) temperature intermediate between these two values.

Figure 5 presents images, delivered by an Agema 880 LW camera, of an electric field using a photothermal film of characteristics identical to those used for the theoretical evaluations. A parallel plane wave of frequency $12 \mathrm{GHz}$ illuminates the film, whose strips are vertical.

In figure 6 (left), the experimental maximum and minimum temperatures along a line perpendicular to the strip, $T^{+}$and $T$, are compared to the theoretical ones. The experimental values of $T^{+}$are near the theory and the values of $T^{-}$are higher. This could be due to the integration effect due to the limited resolution of the cameras. This could explain why the points from the Agema 880 are nearer from theory than those of the 782 camera.

Figure 6 (right) presents the same type of comparison for the $\mathrm{x}$-integrated temperature, here called $T_{\text {integr }}(\omega):(1 / \Pi) \int_{-\pi / 2}^{\pi / 2} T(x, \omega) d x$. The experimental integrated temperature is higher than the theoretical one. This is due to the lateral heat diffusion in the film, from the absorbing strip to the non absorbing ones.

The experimental calibration curve $T_{\text {integr }}(f)$ is to be used when the camera space resolution is lower than the space period of the film strips. On the contrary, for high resolution allowing to distinguish the hot absorbing strips from the cold ones, the $T^{+}(f)$ calibration curve must be used in conjunction with the maximum temperature measured in the hot strips.

\section{Application to the analysis of the field in the vicinity of the end of a wave guide}

To illustrate the possibilities of the technique, we present here the analysis of the near field in the vicinity of the end of a Ku open guide. For this purpose, a film of higher anisotropy is positioned in the $(x, z)$ plane of symmetry of the guide (see Fig. 7 ). The absorbing strips have the same geometrical characteristics than those of the previous film, but their square resistance is $R=181 \Omega$, leading to an anisotropy factor of 200. They are successively put in vertical and horizontal positions for analysing respectively the $E_{z}$ and $E_{x}$ field components. The IR camera used is an Agema 782 LWB, viewing a field $3 \mathrm{~cm} \times 3 \mathrm{~cm}$ thanks to a $12 \mathrm{~mm}$ extension ring. The EM field at $12 \mathrm{GHz}$ is amplitude modulated at the frequency of $20 \mathrm{~Hz}$.

The results are presented in Fig. 8 and compared to the numerical results of a finite difference time domain code (ALICE) in Fig. 9. This figure gives the $x$ - and $z$-distributions of both $E_{X}$ and $E_{Z}$ components of the near field. The z-distributions correspond to a x-abscissa of $2.5 \mathrm{~mm}$ from the guide aperture, and the $x$-distributions correspond to the centre line of the guide for the $E_{Z}$ component and to the horizontal upper plane of the guide for the $E_{X}$ component. A good agreement between theory and experiment is found, in particular the decrease of the fields with increasing distances from the guide aperture. The code does not take into account the thickness of the guide wall $(1 \mathrm{~mm})$. This could explain some discrepancies, in particular the fact that the calculated $E_{X}$ field z-extension is wider than the measured one. 


\section{Conclusion}

The feasibility of manufacturing well-controlled, highly-anisotropic photothermal films has been demonstrated. The ability of these film to characterise by the EMIR technique the vectorial components of electromagnetic fields is proved by EMIR measurements of the near field in the vicinity of an open guide and comparison to the results of a finite difference code.

\section{References}

[1] BALAGEAS D. and LEVESQUE P., "EMIR : a photothermal tool for electromagnetic phenomena characterization", Revue Générale de Thermique, 37, 1998, p 725-739

[2] LEVESQUE P., DEOM A., and BALAGEAS D., "NDE on absorbing materials using microwave stimulated infrared thermography ", QIRT'92, Editions Européennes Thermique et Industrie, Paris, 1993, p 302-307

[3] NACITAS M., LEVESQUE P., and BALAGEAS D., "Lock-in infrared thermography applied to the characterization of electromagnetic fields", QIRT'94, Editions Européennes Thermique et Industrie, Paris, 1995, p 286-292

[4] BALAGEAS D., LEVESQUE P., NACITAS M., J.-C. KRAPEZ, and GARDETTE G., "Microwaves holography revealed by photothermal films and lock-in IR thermography: Application to NDE of dielectric and radar absorbing materials", QIRT'96, Edizioni ETS, Pisa, 1997, p 73-78

[5] LEVESQUE P. and BALAGEAS D., "Single-sided interferometric EMIR method for NDE of radar absorbing structures", QIRT'98, Akademickie Centrum Graficzno-Marketingowe Lodart S.A., Lódz, 1999, p 36-42.

[6] BOUCHITTE G. et PETIT R., Radio Science 24-1, 1989, p13-26

\begin{tabular}{|c|c|l|c|c|c|}
\hline$f \mathrm{~Hz}$ & $\mathrm{~T}^{+} /\left(\mathrm{P}_{0} / 2 \mathrm{~h}\right)$ & \multicolumn{1}{|c}{$/\left(\mathrm{P}_{0} / 2 \mathrm{~h}\right)$} & $\mathrm{T}_{\text {integr }} /\left(\mathrm{P}_{0} / 2 \mathrm{~h}\right)$ & $\left(\mathrm{T}^{+} / \mathrm{T}^{-}\right) \mathrm{dB}$ & $\left(\mathrm{T}^{+}-\mathrm{T}^{-}\right) / \mathrm{T}_{\text {mean }}$ \\
\hline 0.5 & 0.050 & 0.0088 & 0,0287 & 4.2 & 0.45 \\
\hline 1 & 0.033 & 0.0068 & 0,0157 & 6.8 & 0.65 \\
\hline 5 & 0.010 & 0.00015 & 0,0031 & 18. & 0.97 \\
\hline 10 & 0.006 & 0.000012 & 0,0015 & 27. & 0.996 \\
\hline 20 & 0.003 & 0.00000048 & 0,0007 & 38. & 0.9997 \\
\hline
\end{tabular}

Table 1 : Theoretical temperature increase modules as a function of the modulation frequency for the film of fig. 1.

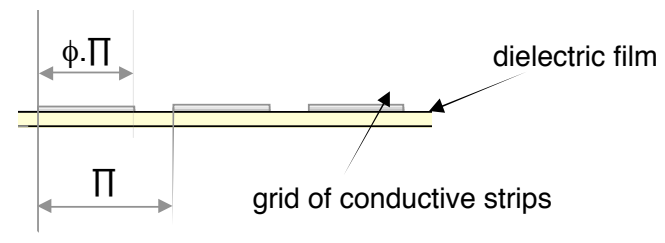

$E / / \otimes H / l$

Figure 1 : Structure of the anisotropic photothermal film 


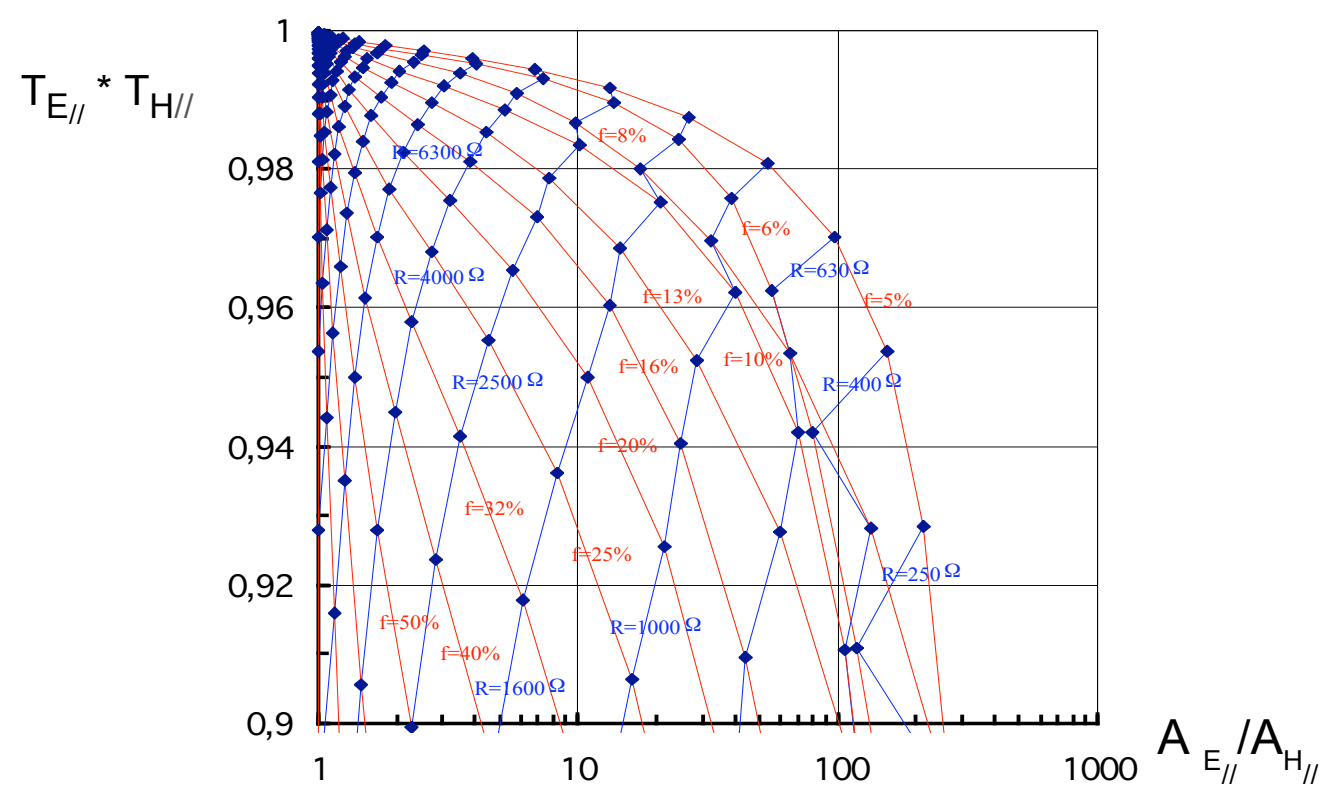

Figure 2: Graph allowing the determination of $\mathrm{R}$ _and $\phi$ (here $\mathrm{f}$ ) of an anisotropic film
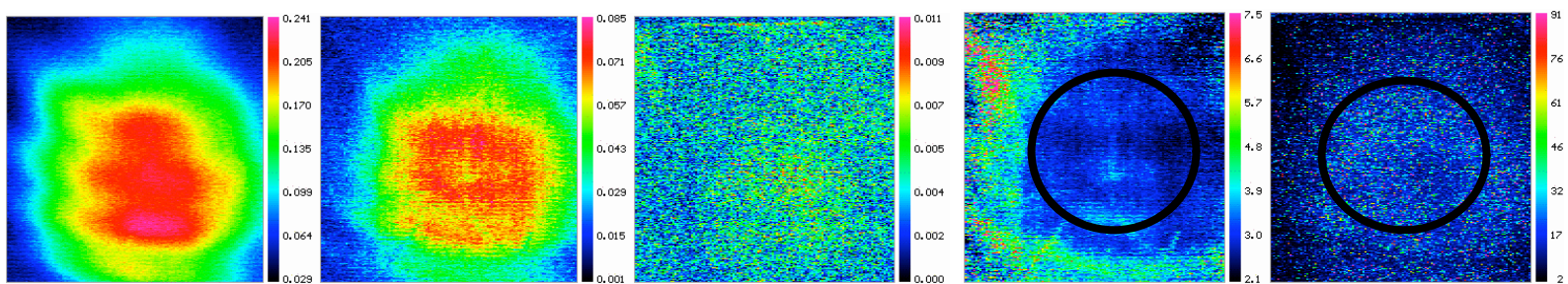

Figure 3 : EMIR images corresponding to the same EM field viewed with (from left to right): isotropic film, anisotropic film // to the E// incident field, anisotropic film perpendicular to this E/l, distribution of absorptivity, distribution of anisotropy factor . Agema $880 \mathrm{LWB}, 5 \mathrm{~Hz}$

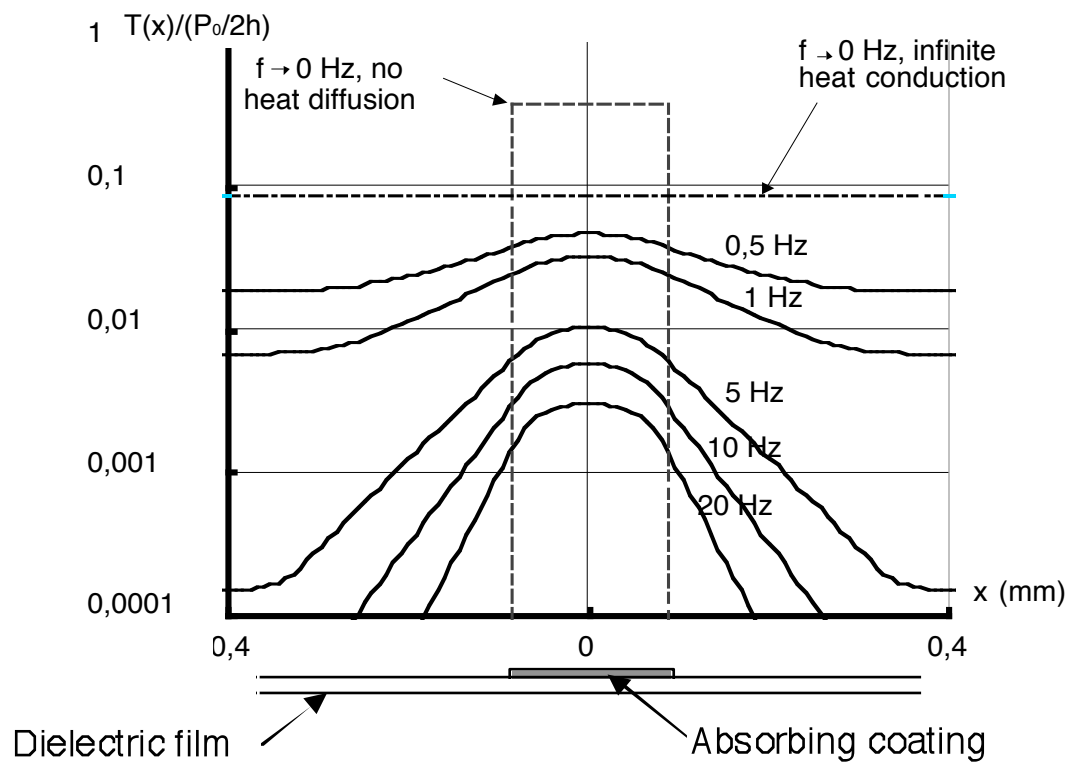

Figure 4: Theoretical normalised temperature increase module of an anisotropic film $\underline{\mathrm{R}}=1020 \Omega, \pi=800 \mu \mathrm{m}, \phi=0.219)$ for various modulation frequencies. 


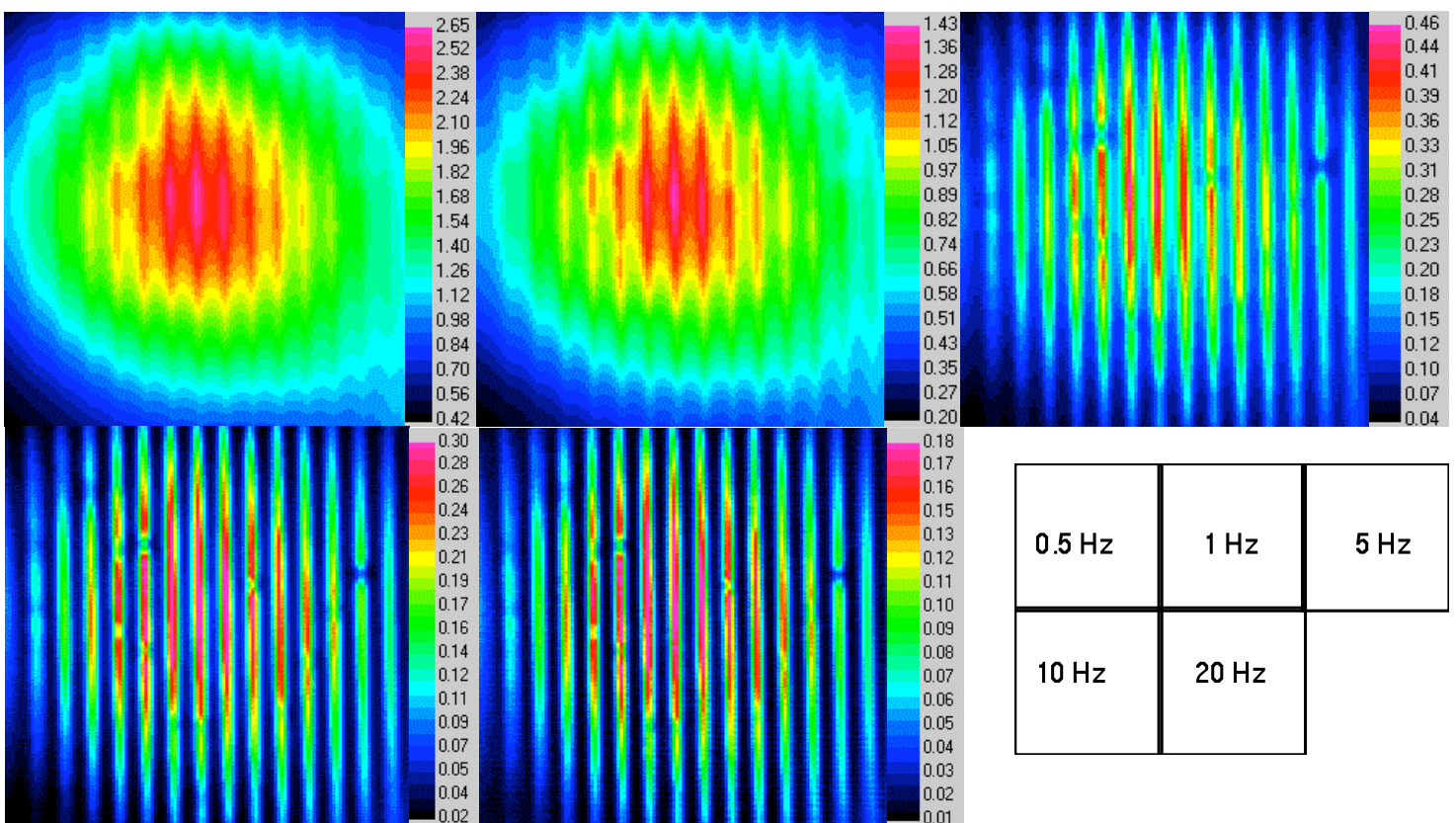

Figure 5: IR images (Agema $880 \mathrm{LWB}$ ) of a field at $12 \mathrm{GHz}$ for various modulation frequencies (film characteristics: $\mathrm{R}=1020 \Omega, \Pi=800 \mu \mathrm{m}, \phi=0.219$ ).

$\mathrm{T}^{+} /\left(\mathrm{P}_{0}^{\prime} / 2 \mathrm{~h}\right), \mathrm{T}^{-} /\left(\mathrm{P}_{0}^{\prime} / 2 \mathrm{~h}\right)$

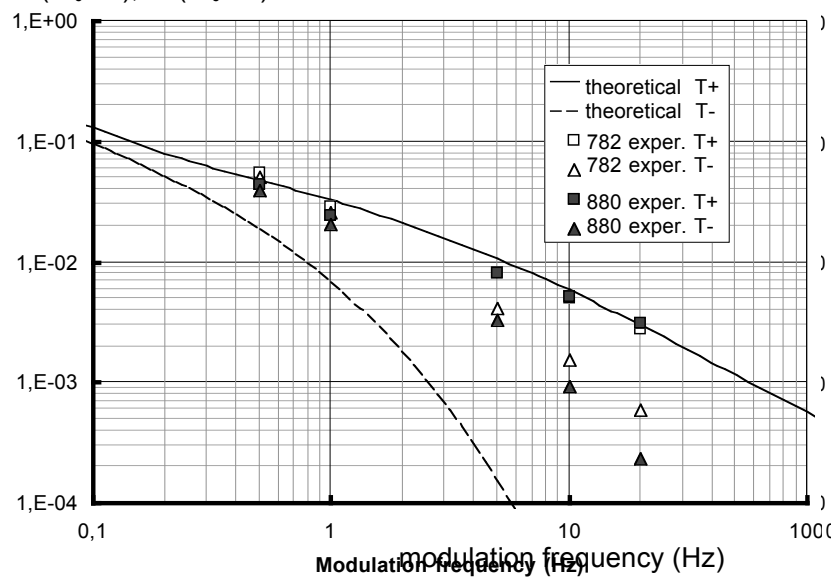

$\mathrm{T}_{\text {integr }} /\left(\mathrm{P}_{0}^{\prime} / 2 \mathrm{~h}\right)$

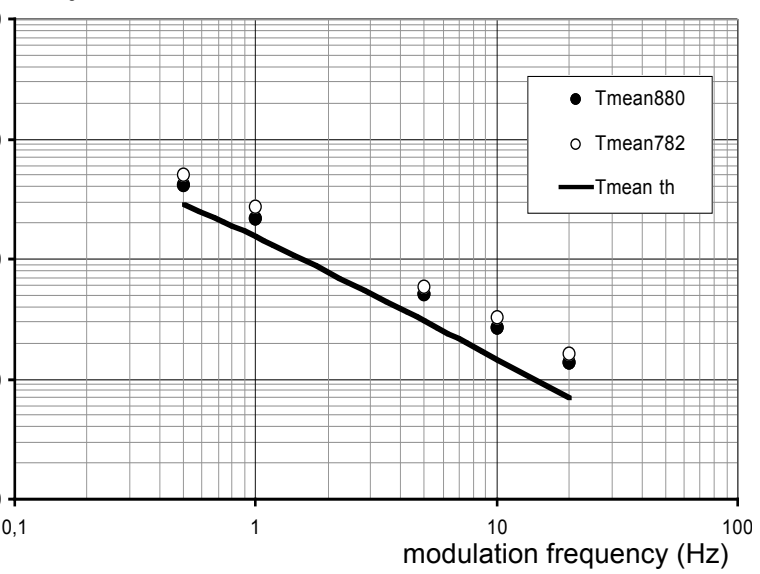

Figure 6 : Experimental and theoretical modules of the modulated part of the film temperature increase: comparison of the integrated (right), maximum and minimum (left) temperatures

$$
E_{z} \text { analysis } \quad E_{x} \text { analysis }
$$
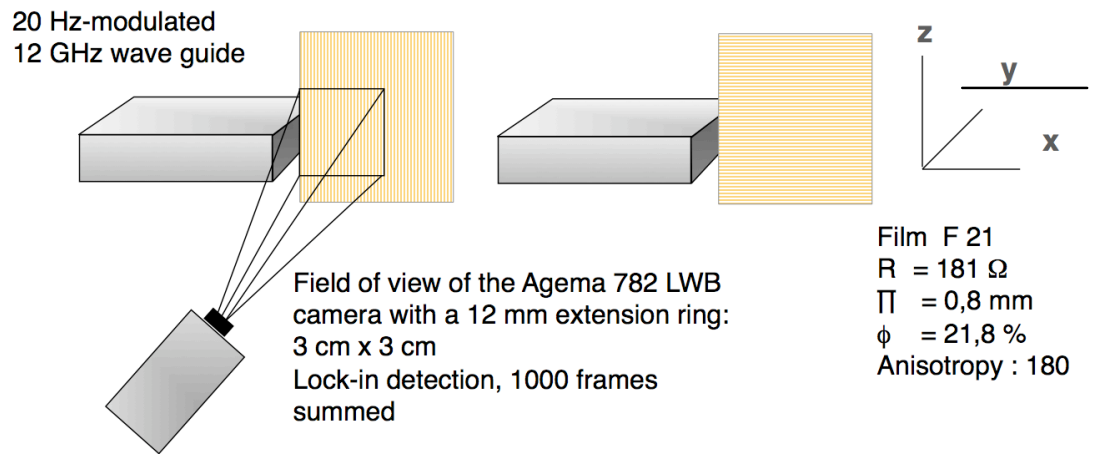

Figure 7 : Experimental configurations for $\mathrm{E}_{\underline{z}}$ (Left) and $\mathrm{E}_{\underline{\underline{x}}}$ (right) determination 

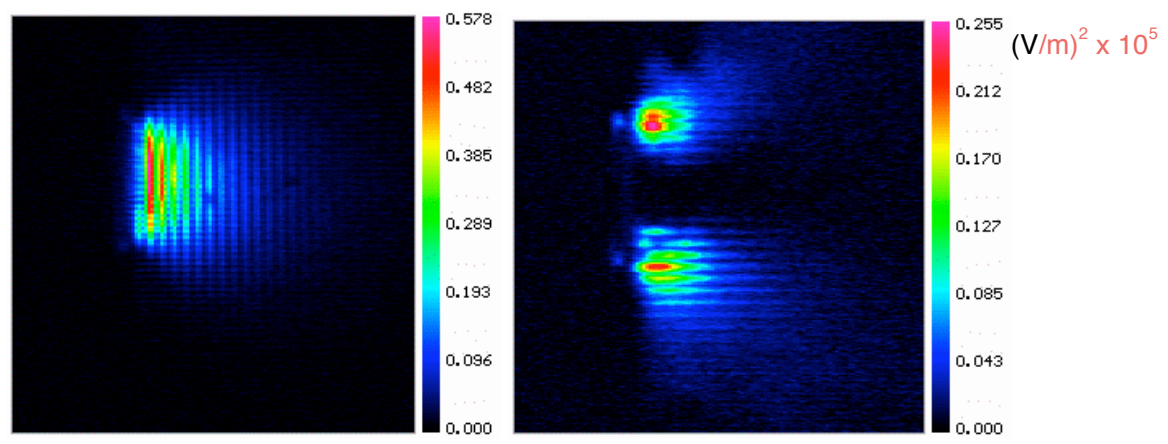

Figure $8: E_{z}{ }^{2}$ (left) and $E_{x}{ }^{2}$ (right) components of the electrical field in the vicinity of the end of the wave guide at $12 \mathrm{GHz}$. Camera used : Agema 728 LWB.
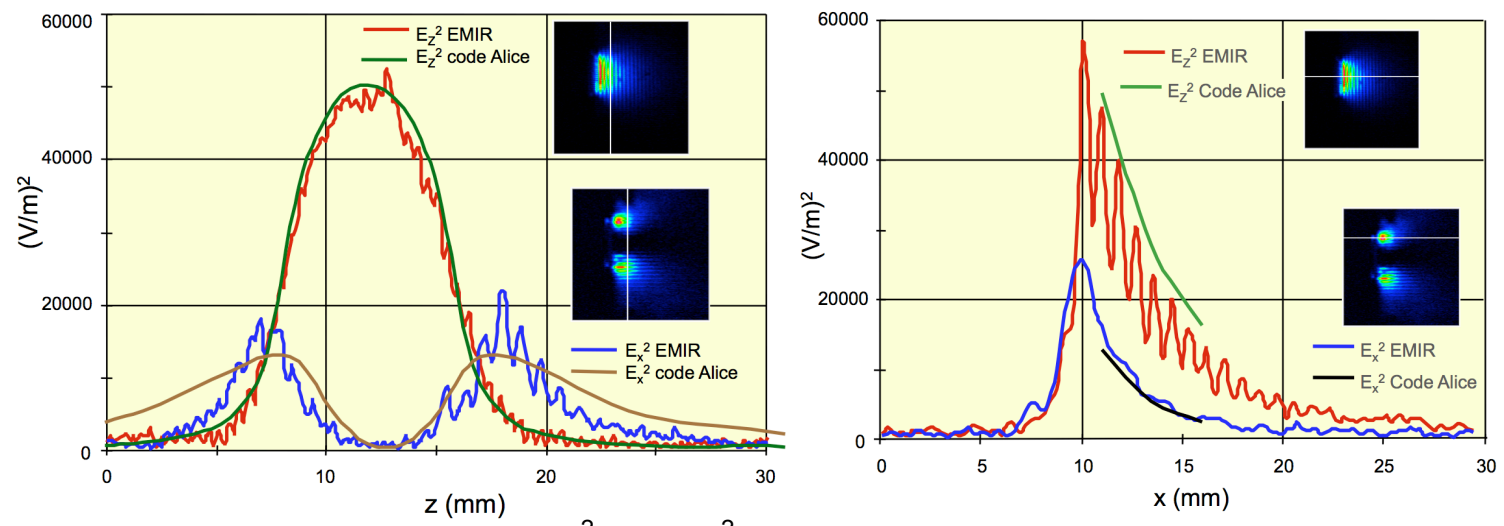

Figure 9 : Left: z-distributions of $E_{z}{ }^{2}$ and $E_{x}{ }^{2}$ fields at $2.5 \mathrm{~mm}$ from the end of the guide and comparison to theory (ALICE code); right: $x$-distributions (from the images of Fig. 8). 